GANIT J. Bangladesh Math. Soc. (ISSN 1606-3694) 38 (2018) 105-118

DOI: https://doi.org/10.3329/ganit.v38i0.39790

\title{
APPROXIMATION OF A COMPLEX GEOMETRIC DOMAIN IN POLAR COORDINATES
}

\author{
Gour Chandra Paul ${ }^{1, *}$, Md. Masum Murshed ${ }^{1,2}$, Md. Mamunur Rasid $^{3}$ and \\ Md. Morshed Bin Shiraj ${ }^{4}$ \\ ${ }^{1}$ Department of Mathematics, University of Rajshahi Rajshahi 6205, Bangladesh \\ ${ }^{2}$ Graduate School of Science \& Engineering Department of Mathematics \& Physics Kanazawa \\ University, Japan \\ ${ }^{3}$ Rajshahi University School, Demonstration Unit-1, IER, University of Rajshahi, \\ Rajshahi 6205, Bangladesh \\ ${ }^{4}$ Graduate School for International Development and Cooperation, Hiroshima University, 1-5-1, \\ Kagamiyama, Higashi-Hiroshima, 739-8529, Hiroshima, Japan \\ "Corresponding author: gcpaul@ ru.ac.bd.com
}

Received 20-08-2018 Accepted 27-11-2018

\begin{abstract}
In this study, a complex geometric domain having a colour picture is approximated through a stairstep representation of the coastal and island boundaries to make it suitable for implementing finite difference method in solving shallow water equations (SWEs) in polar coordinates. As a complex domain, we choose the coastal region of Bangladesh situated at the northern tip of the Bay of Bengal (BOB). To cover the whole coastal region, the pole is selected at the point $O\left(22.96^{\circ} \mathrm{N}, 91.68^{\circ} \mathrm{E}\right)$ in the $r-\theta$ plane assuming it on the mean sea level (MSL). Along the tangential direction, 265 uniformly distributive straight lines are considered through the pole and 959 circular grid lines centered at $O$ are drawn towards the radial direction covering up to $15^{\circ} \mathrm{N}$ latitude in the BOB. Firstly, a matrix with $960 \times 265$ computational grids is constructed from the colour information of the picture. By representing the grids with suitable notations, a proper stairstep algorithm is employed to the matrix obtained with the $960 \times 265$ grids to approximate the coastal and island boundaries to the nearest finite difference grid lines using an Arakawa $\mathrm{C}$-grid system. The whole procedure is done with our developed MATLAB program. The grids representing the coastal stations are also identified closely in the obtained approximated domain. Such a type of presentation of the coastal geometry of the region of interest is found to incorporate its complexities properly with minimum computational grid points.
\end{abstract}

Keywords: Colour picture; Stair-step representation; Coastal complexity; Finite difference method; Polar coordinate system.

\section{Introduction}

Most of the natural phenomena can be modeled by partial differential equations (PDEs) or a system of PDEs. But most of them do not have an analytic solution and hence for solving it, one must rely on different numerical techniques. Finite difference method (FDM) is one of the most popular techniques for solving these PDEs numerically. In this method, the continuous differential 
operators of the PDEs are replaced by the finite difference operators. These difference operators are usually written in terms of grid points, which necessitate a previous discretization of the geometry [1]. However, it is very much difficult to find difference operators for unstructured grids, so the method, in most cases is limited to structured grids. These structured grid systems are used in all the cases of computational studies while imposing the FDM. In the case of studying long wave phenomena originated in shallow water region, shallow water equations (SWEs) consisting of conservation of mass and conservation of momentum are frequently being solved with the help of FDM on a coastal geometry. In most cases, the geometry is found to be complex and irregular and hence it is needed to make the geometry suitable for implementing the FDM. But for incorporating coastal complexities to a considerable accuracy, grid resolution should be high enough, which in turn maximize computational cost. The polar coordinate system is proved to incorporate coastal complexities with minimum cost [7,8]. Thus the solution of SWEs for predicting long waves, namely surges, tides and tsunamis with the aid of FDN in polar coordinates can include several kinds of advantages [7]. But one of the main tasks is the identification and approximation of the coastal and island boundaries as the SWEs are solved on water. There are various ways of approximations. Among them, the stair-step representation is the simplest one [2]. It is of interest to note here that the coast of Bangladesh is familiar for its complexity which lies at the northern tip of the Bay of Bengal (BOB). The tropical storms associated with surges cause a tremendous loss of lives and properties of the coastal people of the country every year, where the coastal complexities have a great contribution [4]. The Shallowness of water, thickly populated low lying islands of different shapes, high tidal range, fresh water and sediment discharge through the world's second largest river system Ganges-Brahmaputra-Meghna etc. make this region the world's most vulnerable one, but the area is insufficiently studied [6]. Thus the coastal region of Bangladesh is chosen as our study area. It is pertinent to point out here that the coastline of Bangladesh is shaped like a funnel. Considering the fact into account, Roy et al. [8] developed a tide-surge superposition model in cylindrical polar coordinates for operational forecasting purpose. But, the model was a stair-step model and the study was conducted using the uniform distribution of grid lines in the radial direction without ensuring fine mesh resolution. But the accuracy of the result obtained from a stair-step model highly depends among other on grid resolution [6]. Also, for accurate prediction of surge associated with a storm, the bottom topographic details of the region, especially for the Meghna estuarine area should properly be incorporated, which necessitates very high resolution of grids [3]. Another important point is to be noted here that the approximation of the coastal and island boundaries was made manually in [8]. Thus the model due to Roy et al. [8] cannot be capable of incorporating coastal and topographic complexities accurately. To solve grid resolution problem, Rahman et al. [8] developed the model of Roy et al. [7] using nested numerical scheme, but the investigation was also limited by the manual representation of the geometric domain. Because manual approximation of such a complex geometric domain must lead to invite an error. Thus the results came out from the investigation of Rahman et al. [7] may be a little bit far from the reality. However, to incorporate the thickly 
populated irregularly shaped big and small islands along with coastal and island boundary curvelinearity and bottom topographic complexities properly, a very fine resolution of grids is required near the coast whereas it is unnecessary away from the coast [5,7]. It is to be mentioned at this juncture that the polar coordinate system itself has the advantages that it ensures high resolution of grids near the pole and lower ones away from it along the tangential direction. Both Rahman et al. [7] and Roy et al. [8] conducted their studies taking this fact into account, where the limitations are the manual representation of the coastal and island boundaries, bottom topography, coastal stations etc. with the use of manual discretizations. Considering the facts into account, in this communication, we intend to develop an efficient way for the discretization of the complex geometric domain through a computer program to make it suitable for implementing finite difference method in polar coordinate system that may help to develop an efficient storm-surge prediction model in cylindrical polar coordinates for the coast of Bangladesh, which in turn can be helpful for the discretization of any funnel-shaped complex geometric domain.

\section{Background}

\subsection{Grid specification}

Grids are defined as smaller shapes formed after discretization of a geometric domain. The digital representation of the geometric domain in matrix form after discretization is termed here as finite difference grid matrix. There are three types of grids namely, structured grid, unstructured grid, and hybrid grid. A structured grid is defined by uniform connectivity. Such a type of grid can be expressed

as an array of two or three dimensions (2D or 3D), which restricts the choice of elements to quadrilaterals in $2 \mathrm{D}$ or hexahedra in $3 \mathrm{D}$. The regularity of the connectivity allows researchers to conserve space as neighborhood relationships are defined by the storage arrangement. On the other hand, an unstructured grid can be defined by irregular connectivity and is not readily expressed as a two or three dimensional array in computer memory. The storage requirements for an unstructured grid in comparison with a structured grid can be substantially large since the neighborhood connectivity are stored here explicitly. A hybrid grid contains both structured portions as well as unstructured portions. It is of interest to note here that in a 2D storm-surge model in polar coordinates, there are three types of unknowns namely, the scalar variable $\zeta$ representing the elevation of the surface of the sea, and the momentum variables $v_{r}$ and $v_{\theta}$ representing the $r$ and $\theta$ components of velocity, respectively, and the parameters can be stored at the specific grids with the help of Arakawa grid systems. It should be noted here that in the case of the Arakawa C-grid system, $\zeta, v_{r}$ and $v_{\theta}$ are stored at (even, odd), (odd, odd) and (even, even) grid points, respectively (see Fig. 1).

\subsection{Stair-step representation}

Through stair-step representation, coastal and island boundaries of a coastal geometry are approximated as either along the nearest $\theta$ - directed odd grid lines ( $i=$ odd) so that on that part of the boundary, there are only $v_{r}$-points or along the nearest $r$-directed even grid line $(j=$ even) so that there are only $v_{\theta}$-points on that part of the boundary (see Fig. 1). Thus the boundary of the 
coast is represented by such a stair-step that at each segment there exists only that component of the velocity which is normal to the segment. This is done in order to ensure the vanishing of the normal component of the velocity at the boundary in the numerical scheme. This representation is known as stair-step representation. A fictitious coast with its stair-step representation is shown in Fig. 1. The stair-step representation obtained in the study is on the basis of the following theorem.

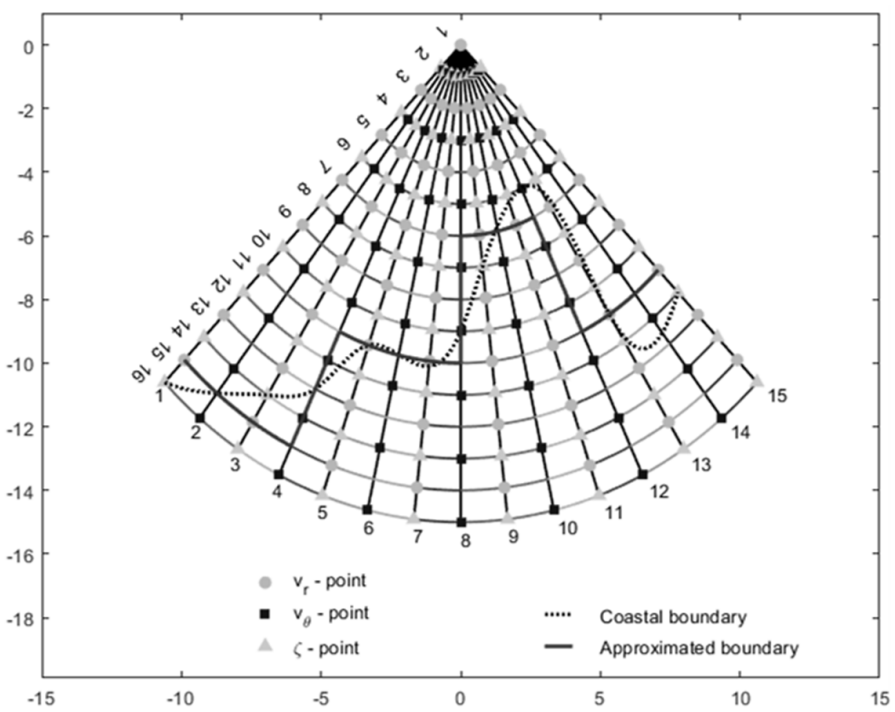

Fig. 1: A fictitious coastal or island boundary and its stair-step representation on a C-grid used in the study.

Theorem 1. If $(i, j)$ is a corner of a stair in the represented stair-step form of $m \times n$ computational grid matrix with $1<j<n$, then $(i, j)$ must be (odd, even).

Proof of theorem 1. A corner $(i, j)$ of the stair in the stair-step representation must have any one of the four forms as shown in Fig. 2. We will show that such a corner $(i, j)$ is (odd, even). It is mentioned earlier that in the stair-step representation, the coastal and island boundaries are approximated as either along the nearest odd $j$-directed grid lines (i.e., $i$ is odd) or along the nearest even $i$-directed grid lines (i.e., $j$ is even). So that in the odd $j$-directed grid lines there are (odd, odd) and (odd, even) points and in the even $i$-directed grid lines there are (odd, even) and (even, even) points. Therefore, there does not exist any (even, odd) point in any segment of the stair step representation. Now, suppose that $(i, j)$ is not (odd, even), then $(i, j)$ must be (odd, odd) or (even, even). Now, if $(i, j)$ is (odd, odd), then $(i-1, j)$ and $(i+1, j)$ are (even, odd). Since $(i, j)$ is a corner, either $(i-1, j)$ or $(i+1, j)$ must lie on a segment of the stair-step (see Fig. 2). Which is a contradiction. Again if $(i, j)$ is (even, even), then $(i, j-1)$ and $(i, j+1)$ are (even, odd), which is also a contradiction. Therefore, $(i, j)$ must be (odd, even). It is to be noted here that the theorem with its proof can be found in detail in the investigation of Murshed et al. [2] but for understanding the complete notion behind this article without deviating the readers' attention to references, it is presented here also. 


\section{Approximation Procedure}

Firstly, a colour picture of the physical domain covering the area bounded by longitudes $85^{\circ} \mathrm{E}$ to $95^{\circ} \mathrm{E}$ and latitudes $15^{\circ} \mathrm{N}$ to $23^{\circ} \mathrm{N}$ (see Fig. 3) is generated through ArcGIS software. To employ finite difference technique in solving SWEs in polar coordinates based on a domain of irregular shape, it should be approximated with rectangular, square or circular mesh grids, where the curvilinear boundaries are usually approximated through a proper stair-step representation. To make such a representation, the Adobe Illustrator software is firstly used to make the map smooth removing the unnecessary parts. Two colour pictures of that domain are produced by the process mentioned above. One of them is with the three colours, namely blue, white and black representing water, land, and coastal stations, respectively (see Fig. 4), and the other one is with the two colours, namely blue and white representing the same as in the first one.
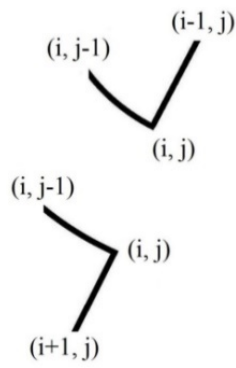

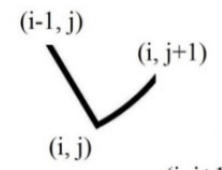

$(1, \mathrm{j})$

(i, j)

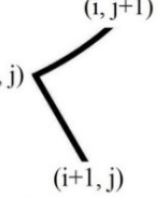

Fig. 2: Positions of corners in different segments found in stair-steps.

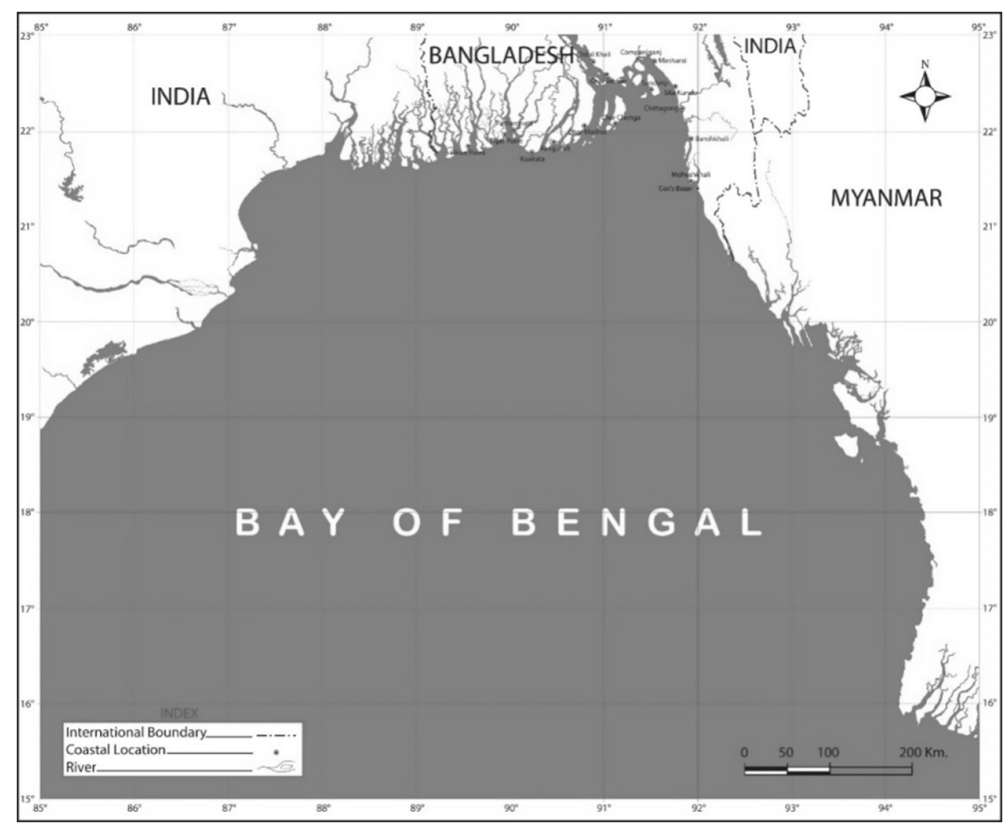

Fig. 3: A colour picture of the northern part of the Bay of Bangle covering the area by longitudes $85-95^{\circ} \mathrm{E}$ and latitudes $15-20^{\circ} \mathrm{N}$ along with some coastal stations 
It is pertinent to pinpoint out here that our whole approximation procedure was made with the aid of our own developed MATLAB program. First, the program is used to read the picture constructed with the blue and white colours. Setting the picture as a background, the pole is selected at the point $O\left(22.96^{\circ} \mathrm{N}, 91.68^{\circ} \mathrm{E}\right)$ on that picture. Then, along the tangential direction, 265 uniformly distributive straight lines are constructed through the pole and 959 circular grid lines with center at $O$ are drawn along the radial direction covering up to $15^{\circ} \mathrm{N}$ latitude in the BOB. Therefore, there are $959 \times 265$ grid points in the numerical scheme excluding the pole. Although all the 265 grid lines meet at the pole, for suitability of numerical treatment, it is assumed that there are 265 distinct grid points at the pole. Thus there are $960 \times 265$ grid points in the computational scheme. Since the pole is situated at the land, no parameters will be computed at the pole and hence no instability will be involved in the computation. Based on the information of colours of the node points of the grids, a matrix of order $960 \times 265$ is generated consisting of the entries, 0 and 1 corresponding to white and blue colours, respectively. This matrix is the digital representation of the physical domain of our interest, where the entry 1 represents water and 0 representsland of the original domain. Since it is quite impossible to show the matrix of order $960 \times 265$ in an under stable form in one figure, the approximation process of a portion (see Fig. 5) is shown and it is continued to show its updated versions in the whole approximation process. The matrix representing the selected portion is shown in Fig. 6. As the element 1 represents water and 0 represents land in that matrix, so it is clear that consecutive 0 and 1 in that matrix mean that either the entry 1 or 0 must represent the coastal or island boundary in the original domain, i.e., if the $(i, j)$ th element in the obtained matrix is 1 and $(i+1, j)$ or $(i-1, j)$ or $(i, j+1)$ or $(i, j-1)$ th element is 0 , then either 1 or 0 represents the coastal boundary of the original domain.



Fig. 4: Smoothed colour picture of the Bay of Bengal region with 17 stations along the coast of Bangladesh. 1. Hiron Point, 2. Tiger Point, 3. Patharghata, 4. Kuakata, 5. Rangabali, 6. Char Madras, 7. Char Chenga, 8. Chital Khali, 9. Char Jabbar, 10. Companiganj, 11. Mirsharai, 12. SitaKunda, 13. Sandwip, 14. Chittagong, 15.Banshkhali, 16. Moheshkhali and 17. Cox's Bazar 


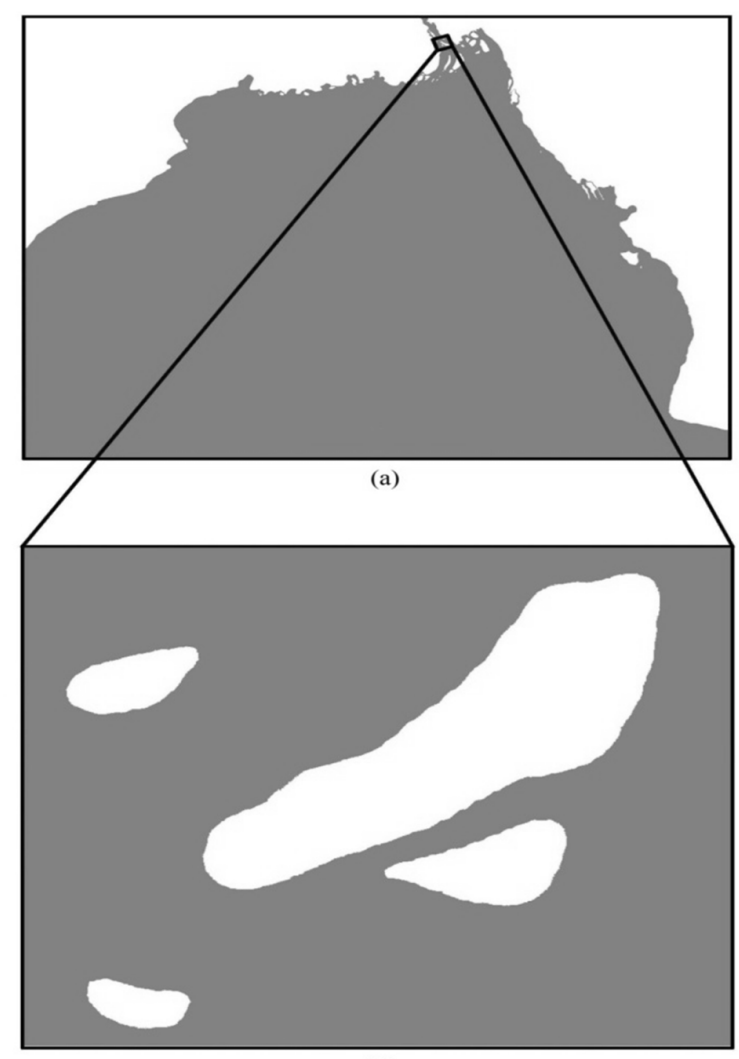

(b)

Fig. 5: A portion (b) of the main picture (a), where white colour represents land, and blue colour represents water.

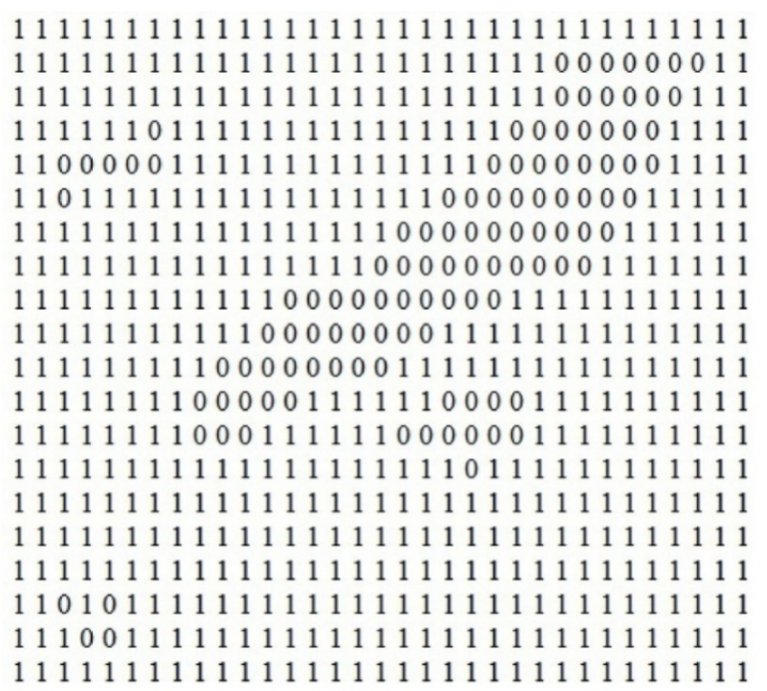

Fig. 6: The matrix of the selected portion, where 0 represents land and 1 represents water. 
The original coastal and island boundaries in the physical domain can be indicated in the obtained matrix by a digit other than 0 and 1 . Here, as in [2], the digit 5 is used to represent the coastal and island boundaries in the obtained matrix replacing 1 by 5 in the above four different cases (see Fig. 7 ). It is clear that the obtained matrix is now composed of 0,1 and 5 representing land, water and the original coastal and island boundaries, respectively. To approximate the coastal and island boundaries through a proper stair-step representation, a matrix (hereafter will be called position matrix) of order $960 \times 265$ composed with the digits $11,12,21$ and 22 corresponding to the positions (odd, odd), (odd, even), (even, odd) and (even, even), respectively, is generated. The positions (odd, even) and (even, odd) are very important in the stair-step representation as the position of a corner of the stair-step representation is always (odd, even) (see Theorem 1), and there does not exist any (even, odd) point in any segment of the stair-step representation (see subsection 2.2). In other words, the stair-step representation can be made through (odd, even), (odd, odd) and (even, even) points. Here, those three types of points near and on the coastal and island boundaries in the obtained matrix are identified and represented by the digit 4 (see Fig. 8) with the help of the position matrix. For instance, if the $(i, j)$ th element is 5 in the obtained matrix and that is not 21 in the position matrix, then 5 is replaced by 4 . Then, the (odd, even) positions are identified in the obtained matrix and represented by 2 based on the logic that if the $(i, j)$ th element is 4 in the updated matrix, and that is 12 in the position matrix, then 4 is replaced by the digit 2 otherwise $(i+1, j)$ or $(i-1, j)$ or $(i, j+1)$ or $(i, j-1)$ th element is replaced by the digit 2 ensuring that the element is 12 in the position matrix (see Fig. 9).

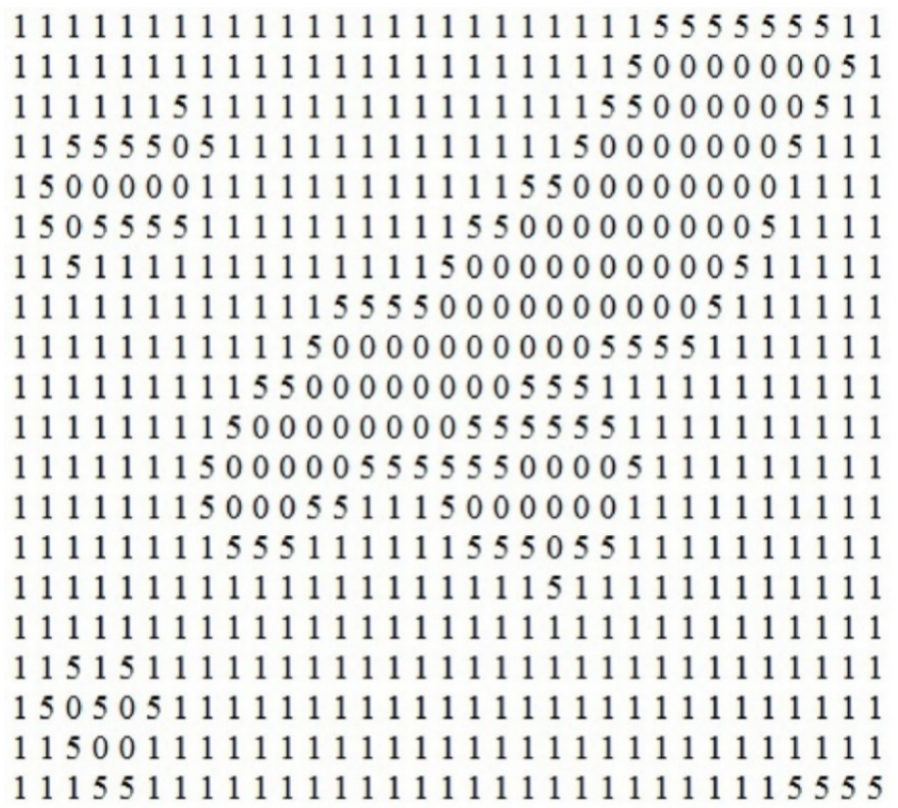

Fig. 7: The updated matrix of the selected portion as shown in Fig. 6, where 0 represents land, 1 represents water and 5 represents coastal or island boundary. 


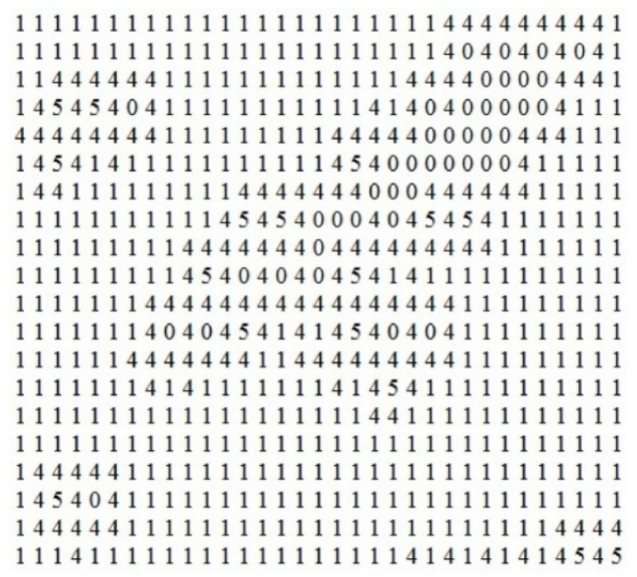

Fig. 8: The updated version of the matrix as shown in Fig. 7, where 0 represents land, 1 represents water, 4 represents water or land near the coast, and 5 represents coastal or island boundary.

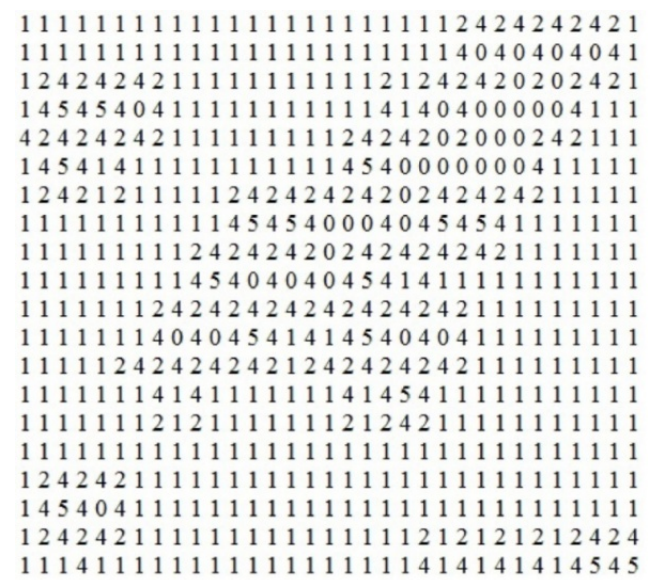

Fig. 9: The updated version of the matrix as shown in Fig. 8, where 0 represents land, 1 represents water, 2 represents approximated coastal and island boundary and 5 represents coastal or island boundary.

Then the middle segment of two corners is identified and is represented by 2 . For instance, if the $(i, j)$ and $(i, j+2)$ th elements are both 2 , then the $(i, j+1)$ th element is replaced by 2 provided that $(i, j+1)$ th element is not 21 in the position matrix and if the $(i, j)$ and $(i+2, j)$ th elements are both 2 , then the $(i+1, j)$ th element is replaced by 2 provided that $(i+1, j)$ th element is not 21 in the position matrix (see Fig. 10). It is seen from Fig. 10 that near the coastal and island boundaries, some square boxes are created with edges formed by 2 surrounding 0,1 and 5 . To remove the square boxes, the land area, and the water area near the coastal and island boundaries are merged with their own region. The technique for this purpose is that if the $(i, j)$ and $(i, j+2)$ th elements are both 0 (or 1$)$ and the $(i, j+1)$ th element is 2 , then the $(i, j+1)$ th element is replaced by 0 (or 1 ), and if the $(i, j)$ and $(i+2, j)$ th elements are both 0 (or 1$)$ and the $(i+1, j)$ th element is 2 , then the $(i+1, j)$ th element is replaced by 0 (or 1 ). Then square boxes surrounding 
the digit 5 are merged with the nearest water region or land region by the same procedure (see Fig. 11). It can be seen from Fig. 11 that a small river is blocked in the updated matrix as indicated by circling 2 (see Fig. 11). This type of problem may happen for other small rivers also. Here, those rivers are connected by the methodology that if the $(i, j)$ and $(i+2, j+2)$ th elements are both 1 , and the $(i+1, j+1),(i, j+1),(i-1, j+1),(i+2, j+1),(i+3, j+1),(i+1, j+2),(i+1, j+3)$, $(i+1, j)$, and $(i+1, j-1)$ th elements are all 2 , then the $(i+1, j),(i+2, j)$, and $(i+2, j+1)$ th elements are replaced by 1 and $(i+2, j-1),(i+3, j-1)$ and $(i+3, j)$ th elements are replaced by 2. A similar work is done for the case where both $(i, j)$ and $(i+2, j-2)$ th elements are 1 , and the $(i+1, j-1)$ th element is 2 . This completes the stair-step representation (see Fig. 12).

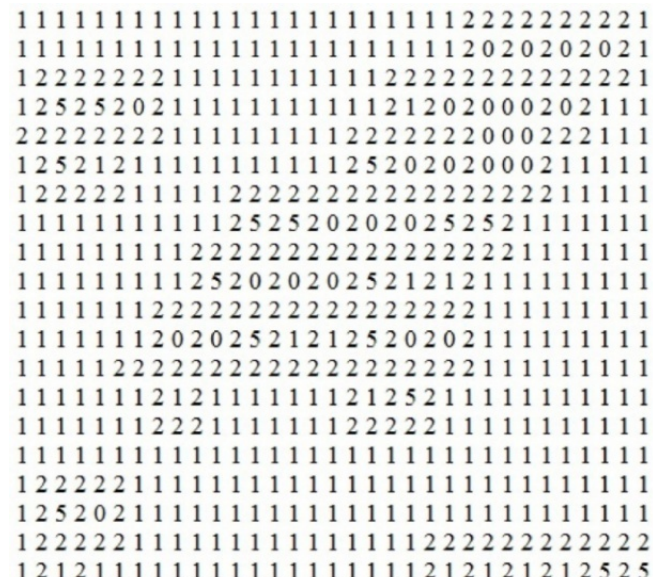

Fig. 10: The updated version of the matrix as shown in Fig. 9, where 0 represents land, 1 represents water, 2 represents approximated coastal and island boundary and 5 represents coastal or island boundary.

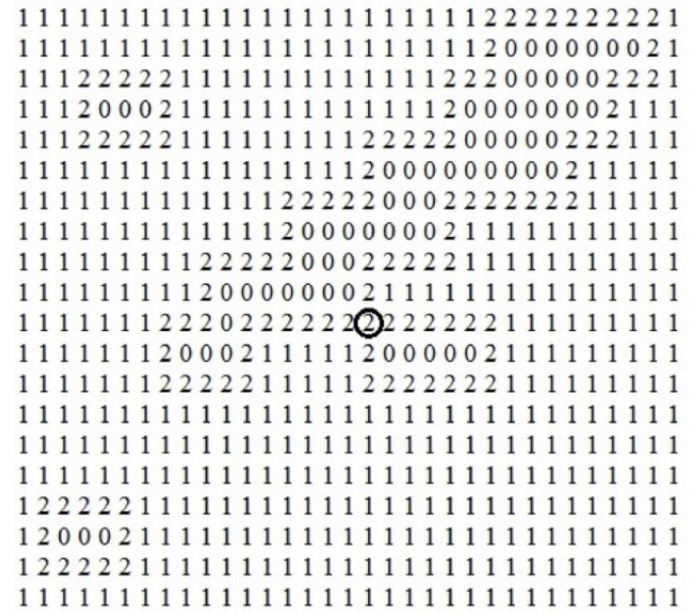

Fig. 11: The updated version of the matrix as shown in Fig. 10, where 0 represents land, 1 represents water, 2 represents approximated coastal or island boundary. 




Fig. 12: The updated version of the matrix as shown in Fig. 11, where 0 represents land, 1 represents water, and 2 represents coastal or island boundary.

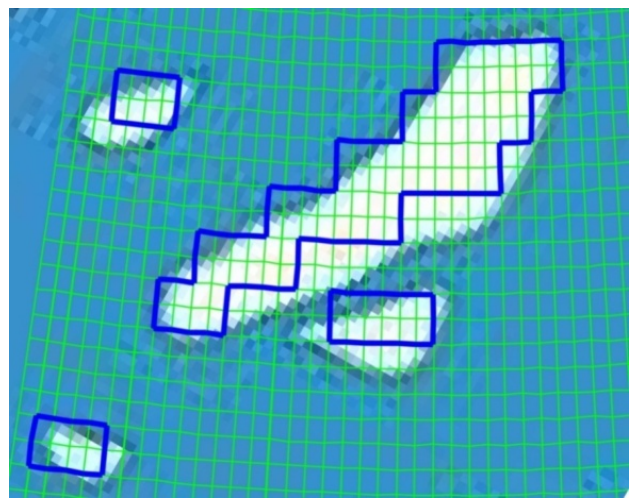

Fig. 13: The representation of land, islands, water and approximated island boundaries of the portion (b) of Fig. 5. This representation is obtained after transforming the matrix shown in Fig. 12 into picture.

Lastly, the positions of the coastal stations are identified. For this purpose, another matrix of the same order $960 \times 265$ as that of the previous one is generated consisting of the entries 0,1 , and 3 from the picture with the respective three colours, namely white, blue and black representing land, water and coastal stations. Thus the digit 3 created in the obtained matrix corresponds to the station locations in the picture is the only difference of it from the previous one. However, the coastal stations are assumed to be located in the water as no calculations are made on the land. If any $(i, j)$ th element in the new matrix is 3 , and the corresponding $(i, j)$ th element in the obtained matrix is 1 , then the $(i, j)$ th position is considered as the location of that specific station for which 3 would be created, otherwise the nearest position, where the digit 1 exists, is considered as the location for that specific station. Our approximated coastal and island boundaries obtained through stair-step along with some approximated coastal stations are presented in Fig. 14, whereas Fig. 15 depicts enlarging versions of some parts of Fig.14 for clear understanding the fact that how accurately the approximations are made. 




Fig. 14: Approximated coastal and island boundaries and some coastal stations, where the asterisks with red colour on the black coloured circles represent the approximated coastal stations.
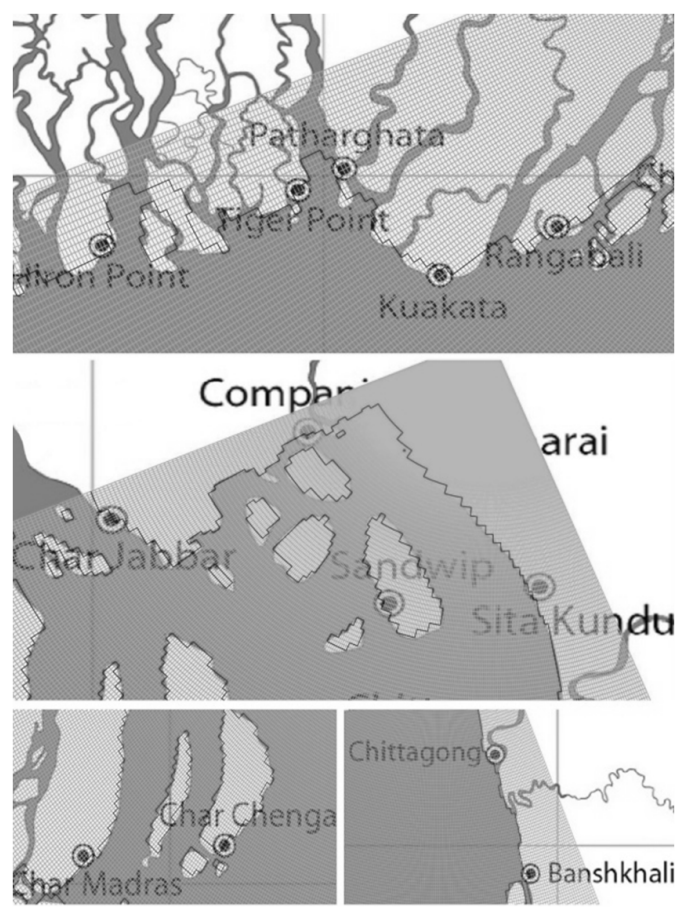

Fig. 15: Enlarge versions of some portions of Fig. 14 representing the approximated coastal and island boundaries and some coastal.

\section{Results and Discussion}

In the present study, a complex geometric domain having a colour picture is approximated through a stair- step representation.It is mentioned that to show our obtained updated matrix with an order of $960 \times 265$ in different steps of the approximation process is quite impossible and hence a 
portion of it is shown and it is continued to show till its final form is obtained (see Figs. 5-13). After completing the approximation, the shapes of the coast and islands in the digital representation are found to be similar to their shapes in the original colour picture when the approximated matrix is transformed into a picture (see Figs. 5, 13). However, to have a clear understanding, our approximated results of the final updated matrix with an order of $960 \times 265$ when transformed into figure are shown in Fig. 14. It can be found from Fig. 14 that the coastal and island boundaries everywhere are approximated with a considerable accuracy. Also, the grids representing the coastal stations are found to be in a reasonable agreement with its original colour picture. For clarifying the fact, some coastal stations are shown in Fig. 15 amplifying the necessary portions of Fig. 14. A point to be noted at this juncture that if the resolution of grids is high enough, then the approximation is not affected much whether the element 1 or 0 is replaced by 5 to represent the original coastal or island boundaries but if the grid resolution is not high enough, then the approximation of small islands or small rivers will be affected whether only 0 or only 1 is replaced by 5 . A solution to overcome this situation may be, for some cases, 0 should be replaced by 5 and for some cases 1 should be replaced by 5 . Since the grid resolution used in the present study is high enough, either 0 or 1 can be replaced by 5 . Resolution of grids can be improved whether necessary and a matrix with grids by the technique can be obtained. The obtained approximated geometry along with its digital representation can be used to solve SWEs by finite difference method or by the numerical method of lines in polar coordinates for simulation/prediction of water levels due to tides, storm surges, tsunamis or any other purposes, which necessitate accurate incorporation of the complexities of the coast. The accuracy of the approximated land-sea interface and coastal station representations can be increased, if necessary, by improving grid resolution.

\section{Conclusion}

A considerable accurate representations of the interface of land and sea, as well as coastal stations along the coast of Bangladesh covering up to $15^{\circ} \mathrm{N}$ latitude in the BOB is made with $960 \times 265$ computational grids in polar coordinate system approximating the coastal and island boundaries through a proper stair- step representation to be compatible of using finite difference technique in solving SWEs in various purposes, namely storm surge, tide, tsunami etc. simulations/predictions. Such a type of presentation of the coastal geometry of the region of interest is found to incorporate its complexities properly with minimum computational grid points, which in turn will reduce computational cost. This method of approximation can be used to approximate any funnel-shaped complex coastal geometry.

\section{Acknowledgment}

This work is partially supported by a grant from the Dean of the faculty of science of the University of Rajshahi, Bangladesh with No. 673-5/52/UGC Project/Science-8/2014, and a grant from the Ministry of Science and Technology of Bangladesh with G.O. No. 
39.009.002.01.00.053.2014-2015/BS-1/ES-34. The authors acknowledge these supports. We would like to thank the anonymous referee for comments and suggestions that helped improve the manuscript. The authors are also thankful to Mr. Md. MahabubAlam, an M.Sc. thesis student, Department of Mathematics, University of Rajshahi, Bangladesh for his help in editing a figure.

\section{REFRENCES}

[1] Kopp, P., Implementation of the characteristic based split algorithm for the shallow water equations in a high order finite element framework. Bachelor's thesis, Technische Universität München. Implementation (2014).

[2] Murshed M.M., Paul, G.C. and Haque, M.R., On the approximation of complex geometric domain to be compatible for the implementation of finite difference method. Int. J. Sci. Eng. Res. 7 (2016), 495501.

[3] Murty, G.S., Flather, R.A. and Henry, R.F., The storm surge problem in the Bay of Bengal. Prog. Oceanogr. 16(1986), 195-233.

[4] Paul, G.C. and Ismail, A.I.M, Contribution of offshore islands in the prediction of water level due to tide-surge interaction for the coastal region of Bangladesh. Nat. Hazards. 65(2013), 13-25.

[5] Paul, G.C., Ismail, A.I.M. and Karim, M.F., Implementation of method of lines to predict water levels due to a storm along the coastal region of Bangladesh. J. Oceanogr. 70(2014), 199-210.

[6] Paul, G.C., Ismail, A.I.M., Rahman, A., Karim, M.F. and Hoque, A., Development of tide surge interaction model for the coastal region of Bangladesh. Estuar. Coast. 39(2016), 1582-1599.

[7] Rahman, M.M., Paul, G.C. and Hoque, A., Nested numerical scheme in a polar coordinate shallow water model for the coast of Bangladesh. J. Coast. Conserv. 17(2013), 37-47.

[8] Roy, G.D., Kabir, A.B.M.H., Mandal, M.M. and Haque M.Z., Polar coordinates shallow water storm surge model for the coast of Bangladesh. Dynam. Atmos. Ocean. 29(1999), 397-413. 